\title{
Ächtung des Selbstlobs und Probleme der Kompetenzdarstellung'
}

Stefan Kihl

Der Begriff der Kompetenz wird in der Pädagogik, Psychologie und Betriebswirtschaftslehre häufig positiv besetzt. Sowohl für kleine Kinder als auch für große Unternehmungen wird die Notwendigkeit von „Kompetenzentwicklung“ betont. Es wird die Bildung von „Kompetenznetzwerken" gefordert, in denen die "Kompetenzpartner" am Ende kompetenter sein sollen als vorher. Unter dem Begriff der ,sozialen Kompetenz" werden so unterschiedliche Merkmale wie „Ernpathiestärke“, „Menschenkenntnis“, „Sensibilität“, „Motivierungsvermögen“" oder „Konfliktfähigkeit" zusammengefasst, und damit suggeriert. dass diese Fähigkeiten notwendig sind, um als Person sich heutzutage zurechtzufinden.

Soziologisch gesehen beschreibt Kompetenz lediglich zugerechnete Zuständigkeit - die Zuständigkeit, einem psychisch Kranken zu helfen, ein schwieriges Problem in einer Verwaltung oder einem Unternehmen anzugehen oder für die öffentliche Ordnung in einer Stadt zu sorgen. Schon die Herkunft von lateinischen Beyriff" "competere" (sowohl "zustehen" als auch "fähig sein") macht darauf aufmerksam, dass man diese Zuständigkeit auf der Basis von zwei Grundlagen erlangen kann: Kompetenz qua Amt und Kompetenz qua Wissen (vgl. Luhmann 2000: 320f).

In der Praxis werden sich ałs zuständig erklärende Leistungserbringer daher immer im Hinblick auf diese beiden Gesichtspunkte beobachtet: „Darf der das?" und ,Kann der das?" Häufig können die beiden Aspekte von Kompetenz zusammenfallen. Eine Managerin hat kraft ihres Amtes als Geschäftsführerin eines Unternehmens eine formal abgesicherte Zuständigkeit für Entscheidungen,

Die Gedanken zur Kompetenzdarsteilung wurden auf der Tagung „Profession, Habitus und Wandel" der Sektion Professionssoztologie im November 2007 an der Humboidl-Universität in Berlin und auf der Abschlusskonferenz des Setninars "Interaktion und Organisation“ an der Universität Bielefeld präsentiert. Eine Readers-Digest-Variante des Arguments ist unter dem Titet „Die verflixte Sache init der Kompetenzdarstellung" (Kühl 2008a) im Einführungsbuch von Uwe Schimank und Nadine Schöneck erschienen. In meinem Buch über Coaching und Supervision findet sich ein Vergleich von Kompetenzdarstellungsschwierigkeiten von Professionen und Nichtgrofessionen inklusives einer Falstudie zur personenorientierten Beratung (Kühl 2008b). 
und diese Zuständigkeit körnte auch dadurch gestützt werden, dass ihre Mitarbeiter sie von ihrem Wissen her als fähig einschätzen, wichtige Entscheidungen zu treffen. Aber häufig ist auch ein Auseinanderfallen dieser beiden Komponenten zu beobachten. Es wird festgestellt, dass ein Berater zwar für zuständig erklärt wurde, aber von seinen begrenzten Fähigkeiten her nicht die ,richtige Intervention" findet. Oder es wird vermutet, dass eine Supervisorin wohl die Fähigkeit habe, eine Problemlage ,aufzuklären", ihr aber nicht die Zuständigkeit dafür gegeben wird (vgl. Luhmann 1964: 278).

Bei beiden Făllen von Kompetenz besteht die Funktion darin, Rückfragen des Gegenübers zu entmutigen (vgl. Luhmann 2000: 204). Man geht davon aus, dass der "Kompetente" selur gute Gründe hat, so zu handeln, wie er handelt. Bei der Entmutigung dieser Rückfragen ist es erst einmal eine zweitrangige Frage, ob diese Gründe durch organisatorische Rollen, ein staatliches Gewaltmonopol oder durch staatliche Lizenzierungen gestützt werden oder aufgrund von angenommenen Wissensvorsprüngen zustande kommen. ${ }^{2}$

Von Vertretern des so genannten dramaturgischen, dramatologischen bzw. inszenierungstheoretischen Ansatzes wird die These aufgestellt, dass Kompetenz zu einem erheblichen Teil auf der Kompetenz in der Darstellung von Konpetenz - auf gut gemachtem Eindrucksmanagement - basiert. Management, so beispielsweise lain L. Mangham (1986), fuße darauf, sich im richtigen Moment als „fachlich fähig" und ,organisatorisch zuständig“ zu präsentieren. Der Kern einer erfolgreichen Beratung, so Timothy Clark (1995), sei die Kunst des „Impression Managements“, die von Unternehmensberatern immer weiter verfeinert werde. Der Kern der Tätigkeit von Professionen wie Ärzten, Juristen oder Geistlichen bestehe, so Michaela Pfadenhauer (2003), in einer ausgeprägten und akzeptierten „Kompetenzdarstellungskompetenz", mit der besonders die fachliche Fähigkeit signalisiert werden soll.

Die Gefahr bei solchen Besclireibungen von Eindrucksmanagement und Kompetenzdarsteliung besteht darin, dass sie zu einer Form von Entlarvungsliteratur verkommen. Überspitzt ausgedruckt: Es wird nicht nur der ironisierende Spruch „There is no business without show business“ (vgl. Neuberger 1990:90) aufgegriften, sondern die Interpretation droht häufig auf die Aussage hinauszulaufen „Business is show business“. Der König trage zwar scheinbar schöne Kleidung, aber in Wirklichkeit sei er nackt. Man müsse nur genauer hinschauen, dann erkenne man dies.

2 Die Anregung, bei der Analyse von Kompetenzen in Anschluss an Luhmann noch systematischer diese beiden Aspekte, also „Kompetenz kraft Amt“ und "Kompetenz kraft Wissen“" zu
unterscheiden und den Hinweis auf dic Funktion der Unterdrückung von Rückfragen verdanke ich Veronika Tacke. Michaela Pfadenllatuer danke ich für Hinweise zum dranatologischen bzw. inszenterungstheoretischen Ansatz.
Dabei wird aber übersehen, dass es in vielen Bereichen notwendig ist, vom Klienten als kompetent wahrgenommen zu werden, um überhaupt seine Kompetenzen anwenden zla können. Jeder, der mit Klienten arbeitet, ist gezwungen, nicht nur kompetent zu agieren, sondern bei den Kunden auch Kompetenzvermutungen zu mobilisieren: Ein Friseur ist darauf angewiesen, seinen Kunden Kompetenz zu signalisieren, damit ihm diese eine möglichst störungsfreie Beschneidung ihrer Haare erlauben. Ein Beratungsteam muss dem Klienten die Sicherheit vermitteln, dass es ein Problem lösen kann - und zwar auch dann, wenn es das erste Mal auf so ein Problem stößt und keine bewährten Routinen für dessen Lösung hat. ${ }^{3}$

Der Arztbesuch (oder, häufig noch schlimmer, der Friseurbesuch) kleiner Kinder macht dies deutlich. Bei Kindern herrscht noch keine Kompetenzvermutung gegenüber den Ärzten (und häufig erst recht nicht gegenüber Friseuren), die Behandlung gestaltet sich deswegen in der Regel schwieriger als bei Erwachsenen. Die Interaktionsbestrebungen der Leistungserbringer sind nicht selten darauf ausgerichtet, mühsam Vertrauen beim Klienten aufzubauen. Es werden Vertrautheitssurrogate in Form der beruhigenden Anwesenheit von Eltern aufgebaut, bevor eine Behandlung einsetzen kann. Aber in ihrem weiteren Lebensverlauf entwickeln sich Kinder dann - jedenfalls meistens - zu Klienten, die den Leistungserbringern mehr oder minder berechtigterweise mit einer Kompetenzvermutung gegenuibertreten.

Berufe, die ihre Leistung am Klienten (oder noch besser in Kooperation mit dem Klienten) erbringen, stoßen auf ein Paradox: Ein Leistungserbringer muss einem Klienten vermittełn, dass er ihm bei seinen Problemen kompetent helfen kann, weil es sonst nur schwer möglich ist, ihm zu helfen. Dass dem Dienstleis-

3 Ich versuche, mich in meiner Argurnentation auf den im angloamerikanischen Sprachraum entstandenen und zurzait im deutschsprachipen Raum als kleines Pflätuchen gedeihenden dramaturgischen", dramatologischer" bzw" inszenienungstheoretischen Ansatz" zu bezie-

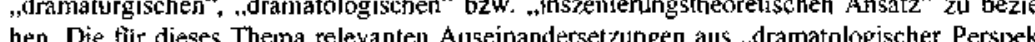

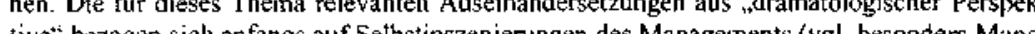
he bezogen sich antangs auf Selbstinszenjeningen des Managemens (vgl. hesonders Mang. ham 1978. Manghan/Overinglon 1987), spater dann besondes auf die Darstellungskompeenzen von Beratern (vgl. besonder Clark 1995, Clark Salaman $1996 a$, 1996), und werden in neterer Zeit im deutschsprachigen Raum vorrangig für dic Untersuchung von Professionen genutzt (vgl. besonders Pfadenhauer 2003; Buer 2004). In diesem auf Inszenierungen ausgerichteten Ansatz wird die Gesellschaft als ein ständiger Strom wechselseitiger Inszenierungen ntit zugnunde liegenden, teilweise beachtlichen dramaturgischen Leistungen der Darsteller beschrieben (vgl. dazu Hitzler 1991; Hitzler 1992). Dahinter steckt die Idee, dass Personen thre Überzeugungen, ihr Können, ihre Einsteltung und ihre Giefühle von ,innen" nach ,außen" bringen müssen und sich dabei inszenieren. Die Stäzke des Ansatzes ist sicherlich, dass durch ihn die „Techniken der Imagepflege“ (Goffman 1986) für sehr unterschiedliche Felder wie die Gothic-Szene, Papsibesuche oder Ärzte-Patienten-Verhältnisse beleuchlet werden. Aber die Gefahr ist - wie bei vielen anderen soziologischen Ansätzen - dass ein interessanter Gedanke auf Expansion gesetzt wird und letztlich dann der Ansprach entsleht, eine ganze Gesellschaftstheorie tragen zu müssen. 
ter eine Kompetenzvermutung entgegengebracht wird, ist deswegen zentral, weil die Leistungserbringung auf das "Gelingen" von einfachen Face-to-FaceInteraktionen angewiesen ist (Luhmann 1972: 270). Eine Lehrerin kann ihren Schislern nur dann etwas beibringen, so schon eine alte pädagogische Binsenweisheit, wenn die Schüler - in ihre Kompetenz vertrauend - mitarbeiten. Eine Psychoanalyse setzt eine Aneinanderreihung gelungener lnteraktionen zwischen Analytiker und Klient voraus. Damit die Interaktion mit dem Klienten gelingt, ist es notwendig, dass dieser die Aneinanderreihung von "Hmms", "Hmms" nich: als Sprachfehler des Analytikers, sondern als konpetente professionelle Gesprächsführung begreift. Auch der Erfolg eines Gesprächs zwischen Supervisor und Supervisand, zwischen Coach und Coachee stützt sich darauf, dass der Klient dem Berater eine Kompetenzvermutung entgegenbringt. Aber wie macht man das? Wie erzeugt man beim Klienten eine Kompetenzvermutung?

\section{Die Schwierigkeiten mit der Kompetenzdarstellung oder weswegen es hăufig besser ist, auf Kompetenzdarstellungen zu verzichten}

Personen versuchen, den Eindruck, den sie bei anderen machen, zu kontrollieren. Gerade bei Leistungserbringern lässt sich beobachten, dass sie versuchen, bei anderen als möglichst kompetent zu erscheinen (vgl. grundlegend aus sozialpsychologischer Perspektive Tedeschi 1981). Bei der Darstellung von Kompetenz gibt es jedoch ein großes Problem: Es löst Irritationen aus, wenn jenand versucht, sich selbst als kompetent darzustellen. Wenn eine Studentin nicht nur gute Leistungen bringt, sondem auch noch offensiv mitteilt, dass sie gut ist, dann kommt es in der Regel genauso schlecht an wie die öffentliche Verkündigung eines Coaches, dass er bereits Top-Führungskräften fast aller $30 \mathrm{DAX}$ Unternehmen geholfen hat. Auch eine mehr oder weniger verkleidete Variante der Kompetenzdarstellung kann ähnliche Effekte erzeugen. Wenn ein Professor auf' seiner Website, in den Klappentexten seiner Bücher oder in den Ankündigungen zu seinen Vorträgen damit wirbt, dass er vom Spiegel einmal als „Zeitmanagement-Papst" bezeichnet wurde, verweist er zwar auf die Kompetenz(oder vielleicht eher Prominenz-) Zurechnung anderer, löst aber allein schon durch die Wiedergabe des Zitats auf seiner (?) Website Irritation aus. Eine personenorientierte Beraterin mag sich von einem Berufsverband die Bezeichnung "Senior Coach" verleilien lassen, gerät aber in Darstellungsprobleme, wenn sie selbst das „Senior“ zu offensiv zu Markte trägt. „So fühlt man Absicht und man ist verstimmt" (Goethe 1999: II Aufzug, 1. Auftritt).

\section{I.I Die Äching des Selbstlobs}

Der Hintergrund dieses Problems ist eine der zentralen Grundregeln von Faceto-Face-Interaktionen: die Ächtung von Selbstlob (vgl. Pomerantz, 1978). ${ }^{5}$ Mit Sprichwörtern wie "Eigenlob stinkt" oder "Bescheidenheit ist eine Zier" ist diese Interaktionsregel auch in das Altagswissen eingegangen. Wir kennen diese Grundregel und die Probleme, die aus ithrer Missachtung entstehen, aus ganz verschiedenen Gesprächssituationen: Der Latin Lover, der allzu offensiv seine Fähigkeiten im Liebesakt preist, weckt bei der potentiellen Geschlechtspartnerin Misstrauen bezūglich seiner realen Fremdbefriedigungskompetenz. Die Verkăufer von Templeton-Fonds, die allzu offensiv ihre UnterschichtHerkunft durch Reichtumssignale zu kaschieren suchen, erwecken bei potentiejlen Käufern Zweifel angesichts dieser offensiven Zurschaustellung von Auto, Uhr und Anzug. ${ }^{6}$

Bei dem Problem der dargestellten Kompetenz ist es zweitrangig, ob der Leistungsanbieter "in Wirklichkeit" kompetent ist oder nicht. Altein die "mitgeteilte Kompetenz" macht misstrauisch. Der Latin Lover mag im Bett eine Kanone sein, aber das Herausstellen dieser Fähigkeiten im Vor-Vorspiel reduziert die Wahrscheinlichkeit, dass er dort auch landen wird. Die Chirurgin mag zu den Koryphäen ihres Feldes gehören, aber allein eine überzogene Darsteflung von Kompetenz macht einen Patienten, wenn er denn noch wach ist, misstrauisch. Ein Senior-Supervisor mag bereits eine Reihe von Vorstandsnitgliedern in DAX-Unternehmen aus persönlichen Krisen geführt haben, aber es entsteht Irritation, wenn er dies mit geschwollener Brust auf einer Konferenz mitteilt.

Das Problem liegt darin, dass ein Wortbeitrag, eine Geste oder ein Symbol zu offensichtlich instrumentell eingesetzt wird, um die eigene Kompetenz darzustellen. Man kennt dies aus ganz alltäglichen Situationen. Man sagt etwas,

4 Für Hinweise auf die Soziologie des Selbstlobs und eine anschauliche und höchst amüsante empirische Scliilderung des Kompetenzdarstellungsproblems von Fonds- und Versicherungsverkäufèn danke ich André Kieserling.

5 Arika Pomerantz (1978) nennt dies „self-praise avoidance rule“. Siehe seht fiüh schon Lubmann 1964: 347. Luhtmann verwendet später den besser an seine Gesellschaftstheorie anschlussfähigen Begriff des "Selbstbefriedigungsverbots"; siehe Luhmann 1997: 38If. Die Problematik des Selbstlobs wird auch im dramatologischen Ansatz angesprochen (vgl. Pfadenhauter 1999: 275)

6 Dass dies in Ausnahmefällen auch mit deutlich höherer Kompelenzdarstellungskompeten gelingen kann, zeigt der Fall Jürgen Harksen, der in den 90 er Jahren des letzten Jahrhunderts als Antageberater in Hamburg Kapitalanlegern urn die 100 Millionen DM abgenommen hat (siehe auch Harksen 2006). 
aber letztlich will man etwas ganz anderes als das Gesagte zum Ausdruck bringen. Der Berater meldet sich in einem Workshop zu Wort, nicht weil er etwas zu sagen hat, sondern weil er vermutet, dass von ihm jetzt ein kluger Beitrag verlangt wird.

In der Interaktionspraxis wird häufig unklar gehalten, ob es neben dem mitgeteilten lnhalt noch eine versteckte Mitteilung gibt. Der Berater mag einerseits das Ziel verfolgen, die Diskussion mit einem ganz wichtigen Gedanken voranzubringen, andererseits spielt aber auch die Erwartung, dass er in dieser Situation etwas beizutragen hat, eine wichtige Rolle dafür, dass er sich zu Wort meldet. Jede Kommunikation wird vom Empfänger daraufhin gescannt, ob nicht eventuell etwas anderes mitgeteilt wird.

\subsection{Kompetenzdarstellung über indirekte Kommunikation}

Wegen der Ächtung des Selbstiobs beobachten wir Kompetenzdarstellung meistens in der Form indirekter Kommunikation. Fast alle sprachanalytischen, psychologischen und soziologischen Theorien stimmen darin uberein, dass Kommunikationen nicht nur in direkter, sondern auch in indirekter Form auftreten können. Während direkte Kommunikation eine Form von verbindlich zu verstehender Kommunikation ist (,ich sage, was ich will"), handelt es sich bei indirekter Kommunikation um eine Form der Kommanikation, von der sowohl Empfänger als auch Sender bestreiten können, dass sie stattgefunden hat ${ }^{8}$ Durch den „unterschwelligen Sinn ausdrücklicher Mitteilungen“, „durch Betonung“, „durch Wahl zweideutiger Begriffe“, durch „aufschlussreiches Zögern“, "wohlgezielte Pausen" und "durch Nichteingehen auf Anregungen" werden Informationen gesendet, aber wenn der Empfänger den Sender auf diese Infor-

7 in der Soziologie gibt es die gut eingeführe Unterscheidung zwischen expressiven und instrumentellen Komponenten der Kommunikation (vgl. fur die Anwendung in der Analyse

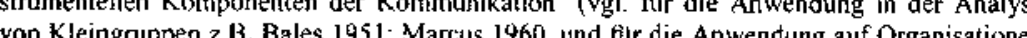
von Kleingnuppen 2. B. Balss 195, Manus 1960, und fur die Anwendung auf Organisationen z.B. Elzioni kation in der Form prasentien, in der es um die Erreichung von Zielen geht: Man fragt, um eine Information zu bckommen, man gibt einen Kommentar ab, um etwas klarzustellen, man liefert eineps Redebeirtag ab, un zusätrliche Informationen in die Debatte zu bringen. Bei expressiver Kommunikation dagegen hat dic Kommunikation nicht die funktion, einen übergeordnelen Zweck zu erreichen, sondern dient dem unmittelbaren Ausdruck von Gefühlen, Wünschen und Bedürfnissen. Wenn man diese Cinterscheidung hier nutzen woilte, müsste man die Kompetenzdarstellung über den Hinweis auf ein eigenes Buch als instrumentell bezeichnen, den selbstlosen Verweis als expresșiv. Mir scheint es jedoch sinnvoller, diese beiden Aspekte als zwei unterschiedliche Formen instrumentellen Verhaltens zu betrachten.

8 Das Phänomen der direkten Kommunikation wird auch als analoge Kommunikation oder als denotative Möglichkeit bezeichnet wird, während indirekte Kommunikation auch als digitale Komınunikation oder als konnotative Möglichkeit benannt witd (vgl. Kieserling 1999; 148ff.). mationen anspricht, kann dieser sich arglos geben und die Intentionen bestreiten (vgl. Luhmann 1964: 364). ${ }^{9}$

Wenn Kompetenzen dargestelit werden sollen, dann können sie eigentlich nur in Form von indirekter Kommunikation dargesteilt werden. Und dabei gilt die Regel: Je indirekter die Kommunikation, desto besser. Das Misstrauen gegenüber dargestellter Kommunikation entsteht, wenn die Kommunikation sich als eine Kommunikation präsentiert, „die meint, was sie sagt“, der Empfänger aber den Eindruck hat, dass noch etwas ganz anderes mitgeteilt werden soll. Der Hinweis auf das gerade erschienene eigene Buch wird nicht dankbar ais Hinweis auf zusätzliche Informationsquellen verstanden, sondern misstrauisch als Darstellung eigener Wichtigkeit wahrgenommen und noch verstärkt, wenn im Klappentext des gekauften Buches der Autor sich als "Coach im Top-Management (sic!) bei internationalen (sic!) Firmen", „brillanten Berater" und ,einer der bedeutendsten Forscher, Modell- und Methodenentwickler" im Bereich der Beratung präsentiert. Das Aushängen von Diplomen ist sicherlich eine eher ungeschickte und auch nur im angloanterikanischen Kontext akzeptierte Praxis. Die Hinweise einer Sekretärin, wie gefragt ihre juristisch, medizinisch, seelsorgerisch, therapeutisch oder beraterisch aktive Chefin ist, belasten eine Interaktion weniger, als wenn es die Chefin selbst vermittelt.

\subsection{Entscheidend ist die Wahrnehmung beim Empfänger}

Für die Interaktion ist es zweitrangig, ob dem Leistungserbringer klar ist, dass er gerade dabei ist, seine Kompetenz darzustellen. Wichtig für die Interaktion ist, wie eine Mitteilung beim Empfänger ankommt. In Gesprächen wird mit Hilfe von Nebenbemerkungen, Betonungen oder kleinen Zeichen von beiden Seiten austariert, worum es genau geht. Dabei wird in der Regel danach gestrebt, die andere Seite nicht in eine unangenehme Situation zu bringen.

Wenn beispielsweise der Empfänger seine Irritation angesichts der zu offensichtlichen Bemühungen um Kompetenzdarstellung zum Ausdruck bringen würde, wäre eine betretene oder gar aggressive Reaktion auf Seiten des Kompetenzdarstellers zu erwarten. Eine Aussage, dass der andere doch bitte nicht zu sehr aufsclnneiden soll, oder ein zu offensichtlich genervter Blick angesichts der

9 Man kann sich die Unlerseheidung zwischen direkter und indirek1er Komınunikation am Kommunikationstegrifi deutlich machen. Kommunikation besteht - systemcheoretisch gesprochen - aus drei Elementen: Information (welche Information wird verrnittelt?). Mitteilung (wie wird die information vermittelt?) und Verstehen (wie wird die Information verstanden?). Direkte und indirekte Kommunikation unterscheiden sich jetzt darin, wie eine information (z.B. die eigene Kompetenz) vermittelt wird: Bei der direkten Kommunikation kann der Sender gegenüber dem Empfänger nicht testreiten, dass die Information mitgeteilt worden ist. Bci der indirekten Kotnmunikation kann der Sender die Mitteilung bestreiten, aber auch der Empfänger kanta so tun, als ob er sic nichs verstanden hat. 
Schilderung eines erfolgreichen offentlichen Auftrittes eines Soziologen, eines Managers oder eines Beraters in vier verschiedenen Ländem kann zu einer nur noch schwer zu beherrschenden Interaktionskrise führen. Das Thema sind dann nicht mehr die erfolgreichen Auftritte in Dänemark, Schweden, Norwegen und Finnland, sondern es verlagert sich zu der häufig konfliktuös geführten Diskussion der Frage, ob und weshalb der vermeintliche Kompetenzdarsteller sich gezwungen sieht, seine Kompetenz so penetrant darzustellen oder - als Verteidigungsreaktion - weshalb der Empfänger eine Schilderung so missverstehen konnte.

Aber diese Interaktionskrisen sind auch bei offensiv dargestellter Kompetenz eher selten. Der Takt verlangt vom Klienten in der Regel, über unglaubhafte Kompetenzdarstellungen hinwegzusehen und den anderen im Interesse der Fontführung des Gesprächs in seiner Selbstdarstellung zu unterstützen. Statt deuthich zum Ausdruck zu bringen, dass einem die Autschneiderei lästig ist, wird freundlich und unterstützend genickt. Man ignoriert die kompetenzdarstellende Komponente in der Kommunikation und behandelt den Hinweis des Coaches auf sein neues Buch als Information, oder man bricht angesichts der Schilderung der erfolgreichen Auftritte in den vier skandinavischen Ländern in große Bewunderung aus.

Dabei ist diese Form des Taktes gegenüber Personen, von denen man abhängig ist (interaktionell zu pflegende Chefs, wichtige Kunden, neue Schwiegerettern), wahrscheinlich. Aber die freundliche Stützung der Kompetenzdarstellung lässt sich häufig auch bei Personen beobachten, zwischen denen eine Machtbalance herrscht. Vielleicht wird durch ein indirektes Zeichen - durch Körperhastung, einen Blick oder ein Lächein - eine Irritation angesichts der Kompetenzdarstellung zum Ausdruck gebracht. Sehr selten wird es dazu kommen, dass der Klient den Leistungserbringer direkt darauf anspricht (zu Takt altgemein siehe Goffman 1983: 212ff.; zu Takt in Organisationen siehe Luhmann 1964: 358).

\subsection{Das Paradox der Kompetenzdarstellung}

Aufgrund der Ächtung des Selbstlobs kommt es zu einem Paradox der Kompetenzdarstellung. Je weniger ein Leistungsanbieter darauf angewiesen ist, seine Kompetenz als Kompetenz zu bewerben, desto höher ist die Wahrscheinlichkeit, dass er beim Empfänger auch auf eine Kompetenzvermutung triff. Eine Künstlerin, die allseits gefeiert wurde und deswegen Bescheidenheit zelebrieren kann, triff nicht nur wegen ihrer Reputation - des Lobes anderer - auf Kompetenzvermutungen, sondern auch deswegen, weil sie nicht durch aggressive Vermark- tung ihrer Kompetenz Misstrauen bei den Betrachtern ihrer Bilder auslöst. ${ }^{10}$ Eine Organisationsberatungsgruppe, der ein guter Ruf vorauseilt, braucht sich nicht selbst aktiv zu loben und verstärkt dadurch noch die Kompetenzvermutungen bei den Klienten.

\section{Der Nutzen von Professionen: Die Entlastung von der Notwendigkeit der individuell zurechenbaren Kompetenzdarstellung}

Die Notwendigkeit, seine Kompetenzen darzustellen, kann je nach Tătigkeit unterschiedlich veiteilt sein. Eine Polizistin braucht seine Kompetenz zur Nutzung einer Pistole in der Regel nicht unter Beweis zu stellen. Man bringt ihr jedenfalls meistens - schon aufgrund ihrer durch die Uniform signalisierten Zugehörigkeit zu einer Berutsgruppe eine Kompetenzvermutung bei der Nutzung einer Pistole entgegen. Einem Piloten begegnet der Kunde - vorausgesetzt, er leidet nicht unter pathologischer Flugangst - mit einem so hohen Maß an Kompetenzvermutung, dass in der Regel keine zusătzlichen Kompetenzdarstellungen durch den Piloten benötigt werden. "Echte Profis“, so könnte man überspitzt formulieren, brauchen keine Kompetenzdarstellungskompetenz."

\subsection{Vom Vorteil der Profession: Der Nutzen der Kompetenzvermutung}

Die Berufs- und Professionssoziologie hat sich in Berufsprestigestudien immer wieder mit der Frage beschäftigt, welche Berufsgruppen von der Bevölkerung an kompetentesten und vertrauenswürdigsten eingeschätzt werden. ${ }^{12}$ Bei der quantitativen Messung der Reputation von Berufen schneiden die Protessionen fast inmer am besten ab. Wenn gefragt wird, welche Berufsgruppe sie am meisten schätzen und bei wem sie am meisten Kompetenz vermuten, gehören die Professionen der Ärzte, Richter und Anwälte, Lehrer und Geistlichen immer zu den am höchsten bewerteten Berufsgruppen. Auf den hinteren Rängen landen

10 Carina Gruber verdanke ich den Hinweis, dass aber auch Bescheidenheit im Auftrith als Inszenierung ,entlarvt" werden kann und dann Irritation beirn Gegentüber auslös.

11 Ich parasitiere dabei an einer Formulierung Michaela Pfadenhauers (2003), die lautet: „Echte Profis haben Konpetenzdarstellungskompetenz", drehe die Aussage der These jedoch un 180 Grad. Die These Pfadenlauers wird überspitzt ausgedrückt in der 7.usammentassung des Buches in einer Presseerklärung der (Iniversităt Dortmund vom 26.3.2003.

12 Leider ist bei der Formulierung der Fragen in den verschiedenen Berufsprestige-Studien nich eindeutig, ob Prestige. Vertrauenswürdigkeit oder Kompetenzwermutung abgefragt wird. Wie das Beispiel der Prostitution zeigt, kann es vorkommen, dass Konpetenzvermutung und niedriges Berufsprestige kombiniert sein kónnen. Es ist aber - wie das Beispiel der Ärzle oder Lehrer deutlich macht - stehr unwahrscheinlich, dass hohes Prestige und geringe Kompetenz vermutungen zusammen aufireten. 
immer Berufe wie Politiker, Prostituierte oder Manager, die zwar ihre Leistungen in Interaktionen mit ihren Klienten erbringen, aber nicht als Profession etabliert sind. Die Ergebnisse dieser mehr oder minder gut gemachten Reputationsmessungen sind seit den zwanziger Jahren des letzten Jahrhunderts übertaschend stabil und varijeren zwischen den einzeinen Ländern auffällig wenig (vgl. früh schon den empirischen Überblick bei Hodge et al. 1964; siehe zur theoretischen Einordnung Kieserling 2004).

Diese Kompetenzvermutungen gegenüber Professionen werden nicht nur und dieser Gedanke ist zentral - durch konkrete Erfahrungen von Klienten mit Professionsangehörigen generiert. Sie werden vielmehr durch eine Vielzahl von "Institutionen" gestützt, die jenseits der eigentlichen Interaktion zwischen Leistungserbringern und Klienten liegen: das Sich-darauf-Verlassen, dass der Tätigkeit des Professionellen ein standardisierter Verhaltenskodex zugrunde liegt; eine wissenschaftliche Verankerung dieses Verhaltenskodexes; die Gewissheit, dass der Professionelle sich diesen Verhaltenskodex in einer mehrjährigen Ausbildung angeeignet hat, und die Sicherheit, dass sich die Profession in einer Form selbst kontrolliert, die es ermöglicht, Kunstfehler zu identifizieren und zu sanktionieren. ${ }^{14}$

Durch die Verselbstständigung der Kompetenzvermutung haben Professionen „Inszenierungsvorteile“ gegenüber Nichtprofessionen. Die Familienanwäl-

13 Auf die umfangreiche Berufsprestigeforsclung kann hier nicht im Detail eingegangen werden (siehe den Uberblicke bei Nakao (1992) oder die neure Diskussion bei Xueguang 2005). Es gibt sicherlich gruppenspezifische Unterschiede, wie das Prestige von Berufen eingeschätzz wird (vgl. dazu z.B. Guppy/Goyder 1984). Es lassen sich Abweichungen von dem hier beschriebenen Trend beobachten. In neutren Untersuchungen wird deutlich, dass die durch Professionen angeleiteten Benife wie beispielswejse der der Krankenschwester sich in inrem Ranking an den hoch reputierten Leiberuf anschließen \{vgl. für Deutschland Allensbacher Institut für Demoskopie 2005). Auffällig ist, dass in Deutsehland Polizisten in der Reget hoch bewertet werden, wälrend im Gegensatz zu anderen Ländern die Reputation von Offiziezen auffällig gering ist. Diesc Abweichungen ätdert aber nichts an der grundlegenden Beabachtung über das hohe Berufsprestige von Protessionen.

14 Der soziologisch häufig unterbestimnte Begriff der „Institution“ wird hier bewusst in Anführanszeicher grimiliken in die meines Erach ail dessen, was jenseits der hteraktionen statindet. Meines Erach tens ist die Darsteflung von Michaela Pfadenhauer, die viel firt das soziologische Interesse an Kompetenz und Kompetenzdarstellung getan hat, widersprüchlich. Einetseits wird in ihrem Buch über Professionalität ausiührlich der Gedarke entwickelt. dass Professionelle über Kormpetenzdarstellungskompetenz verfügen (müssen), andererseits kann man bei ihr in einem kleinen Hinweis lesen, dass Kompetenzdarstellungen nicht nötig sind für ttwas, was allen Beteiligten selbstverständlich ist (vgl. Pfadenhauer 2003: 114). Hier droht die These von „Professionellen als Kompetenzdarsteiler" (vgl. Pfadenhauer 2003: 115) zurückgenommen zu werden, wenn man Professionen gerade dadurch gekentizeichnet sieht, dass deren Kompetenzglaubwürdigkeit „verselbstständigt" wird. tin braucht der nach Scheidung strebenden Ehefrau nicht erst die zentralen Pa* ragraphen des Familiengesetzbuches aufzusagen, damit sich diese auf ein Arbeitsbündnis einlässt. Der Geistliche kann sich jedenfalls im Erstkontakt auf die Reputation seines Berufsstandes verlassen, und der Klient vermutet erstmal, dass er einigermånen predigen, die Beichte abnehmen und die zu kritischen Lebenslagen passenden Bibelstellen rezitieren kant. Dem Mediziner wird insofern vertraut, als dass man - jedenfalls bei Standardbehandlungen - bereit ist, sich einen Praktiker aus dem Telefonbuch herauszusuchen. Erst bei schwerwiegenderen Eingriffen zieht man dann weitere Quellen wie Empfehlungen durch Bekante oder Gerüchte heran. ${ }^{15}$

Diese Kompetenzvermutung gegenüber Professionen kann man an dem wohl prominentesten Kleidungsstück einer Profession verdeutlichen: dem Arztekittel. Vorsoziologisch wurde der Ärztekittel immer wieder als notwendiges Instrument zur Kompetenzdarstellung einer Profession interpretiert. Der Arztkittel sei, so die auch immer wieder in Cartoons aufgegriffene Vermutung, Ausdruck ärztlichen Standesbewusstseins. Erst im und durch den weißen Kittel werde der Arzt zum Arzt. Aber schon empirisch faillt auf, dass viele medizinische Praktiker keinen Kittel tragen: Psychiater tragen ihn nicht, Kinderärzte legen sehr selten einen an, und Hausärzte tragen ibn immer weniger (siehe hierzu und zum Folgenden die populärwissenschaftliche Darstellung von Murphy 2007).

Wie wenig Ärzte auf eine Kompetenzdarstellung über den Arzlkittel angewiesen sind. lässt sich zur Zeit in einem Realexperiment beobachten. Mediziner in Großbritannien sollen - einer Vorgabe des britischen Gesundheitsministeriums folgend - seit 2008 keine weißen Kittel mehr tragen. Hintergrund: In vielen Staaten haben gerade die Infektionen durch gegen Antibiotika resistente Stämme wie Staphylococcus aureus oder Clostridium difticile in den letzten Jahren stark zugenommen. Neben den Krawatten der männiichen Leistungserbringer sind dabei die Ärztekittel die Hauptüberträger dieser Infektionen, weil sie, so zeigen Studien, seltener gereinigt werden als Alltagskleidung. Statt des

15 Der Standardeinwand gerade aus professoralen Kreisen ist, dass man sich doch selir wohl über Unterschiede bei Medizinem, Atwwilten und Therapeuten bewusst sei und sich vor dem Aufsuchen eines Professionals informiere, w'er der "Beste" seines Faches sei. Hier spielt sicherlich eine Rolle, dass gerade unter Wissenschaftlern die Kontaktmöglichkeiten zu Angehörigen anderer Professionen hoch sind und dariuber ein zusätzliches Selektionskriteriuna besteht. UIrich Oevermann hat in einem gemeinsamen Workshop diese Vermutung geäußer. Aufschlussreich war jedoch ein anschließ̉endes Meinungsbild unter den Sudierenden, in dem ein Großteil von ihnen zum Ausdnsck brachte, dass sie sich ihren Arzt aus dem Telefonbuch heraussuchen würden. Aber das ist - zugegeben - anekdotische Eimpirie. Es sei hier wentigstens darau verwiesen, dass es enpirische Studien zur Arztwahl gibt, die leider jedoch in der Regel keinen Vergleich zur Wahl von aicht als Profession organisierten Leistungserbringern bieten (vgl. zulelzt für den deutschsprachigen Raum z.B. Kriwy/Aumüller 2007). 
Kittels empfiehlt das britische Gesundheitsministerium das Tragen von Hemden mit kurzen Ärmeln oder T-Shirts, die tagtäglich gewechselt werden. Nur wenn mit Blut, Eiter oder Exkrementen hantiert wird, sollte mit Plastikschürze, Einmalhandschuhen und Mundschutz gearbeitet werden, die dann unmittelbar nach der Behandiung entsorgt werden (vgl. Bartens 2007).

Uberraschend ist, wie problemlos sich die Abschaffung des Ärztekittels in Großbritannien durchzusetzen scheint. Mehrere Studien haben gezeigt, dass die hauptsächliche Funktion von Ärztekitteln darin besteht, besonders in Krankenhäusern den Patienten eine schnelle Identifikation des ärztlichen Personals zu ermöglichen (siehe z.B. Ikusaka et al. 1999). Aber gerade dafür gibt es auch andere, weniger infektiöse Möglichkeiten: Namensschilder. ${ }^{16}$

\subsection{Die Notwendigkeit von .,Kompetenzhygiene" durch Professionsangehörige}

Selbstverständlich kann dieser hier nur idealtypisch dargestellte Prozess in der konkreten Interaktion zwischen Professionsangehörigen und ihren Klienten immer wieder gebrochen werden. Wenn ein Mandant durch den Hinweis eines befreundeten Anwalts einen Schnitzer bei der Erstellung einer Urkunde durch seine Notarin entdeckt, führt dies in dem konkreten Fall zu ejner Reduktion de Kompetenzvermutung. Ein Zahnarzt, der für seine Klienten sichtbar nicht die habituelle Selbstverständlichkeit bei der Behandlung von Karies zeigt, sondern regelmäßig bei seiner Arzthelferin nachfragt, was er denn jetzt machen soll, reduziert Kompetenzvermutungen beim Patienten. ${ }^{\text {" }}$

Die Professionellen reagieren auf solche reduzierte Kompetenzvermutung ihrer Klienten häufig mit übermäßig inszenierten Kompetenzdarstellungen, die die Interaktionskrisen dann weiter verschärfen - wenn z.B. junge Ärzte mit einem für sie unbekannten Problem koufrontiert werden, gegentiber dem Patienten häufig mit äbertriebener Kompetenzdarsteflung auttreten. Bei jungen Lehrern, die durch eine Klasse unter Druck gesetzt werden, lässt sich nicht selten beobachten, dass sie die Situation noch dadurch verschlimmenn, dass sie zu

16 in der einzigen mir bekanmien Auseinandersetzung Luhtmanns nit dem Arztkittel sieht er die Funktion vorrangig darin, einerseits ,Statusansprüche, Rollentypen oder Berutszugehörigkeiten (zu) manifestierer und zugleich entsprechende Handlungen" anzukundigen (vgl. Lufmann 1964: 363f.J. Ein grober Teil der empirischen Studien über Árzekittel kommt aus der Medizinwissenschaft. Es wäre sicherlich ein interessantes Forschungsfeld, die Funktion von Ärzlekitteln und die Effekte ihrer Abschaffung soziologisch zo untersuchen.

17 Dabei besteht dann natürlich auch dic Möglichkeit, dass eine Entäuschung mit ejnern Angehörigen einer Profession auf alle Angehörıgen dieser Berufsgruppe übertragen wird und negative Urteile über die Lehrer, die Ärzte oder die Juristen entsiehen (vgl. Luhnaan 1974:3). aggressiv versuchen, ihre Kompetenzen, die von den Schülern in Frage gestelft werden, darzustellen. ${ }^{18}$

Zur Vermeidung von solchen Interaktionkrisen müssen Angehörige von Professionen in ihrer Beziehung zu Klienten "Kompetenzhygiene" betreiben um die Idee des Hygienefaktors von Frederick Herzberg zu paraphrasieren. Kurz: Sie müssen darauf achten, dass ibre Klienten nicht an ihren durch Institutionen abgesicherten Kompetenzen zweifeln. Wenn ein Patient stirbt oder ein Schüler nicht lernt, muss sichergestellt werden, dass dies nicht auf Fehler des Professionellen zurechnet wird, sondern entweder auf ein nicht beeinflussbares Schicksal (,jeder muss mal sterben", „warum habe ich einen so dummen Sohn") oder auf den Klienten selbst (,hätte er doch die Medikamente genommen", „hätte er doch mehr gelernt"). Unterschiedliche Auffassungen über eine Vorgehensweise, die immer wieder in einem Ensemble von Professionelien autreten können, gehören, so die häufig herrschende lnteraktionstegel, nicht vor dem Klienten ausdiskutiert, um nicht Kompetenzzweifel aufkommen zu lassen. Unsicherheiten eines Professionellen in Bezug auf die nächsten Arbeitsschritte werden häufig auf der Hinterbühne unter zur Hilfename der "Klinikleitfaden" geklärt und dem Klienten gleichzeitig eine „Sicherheitsfassade“ geboten.

Die Leistung von Hochstaplern ist, dass sie diese "Kompetenzhygiene" hinbekommen, ohne dass sie die entsprechenden Fachkenntnisse anerzogen bekommen haben und ohne dass sie über lange Jahre den einen professionelten Habitus ansozialisiert bekommen haben. $O b$ man sich die in der Regel hervorragend gemachten Imitationen eines Offiziers durch Wilhelm Voigt (siehe Zuckmayer 1995 für eine romanhafte Aufarbeitung), eines Augenarztes durch Karl May (siehe Hülsman 1969), eines Psychiaters durch Gert Postel (siehe Postel 2001), eines Piloten durch Frank W. Abagnate (siehe Abagnale/Redding 2003) oder die Imitationen des Habitus von Oberschichten durch Anlagebetrüger wie Jürgen Harksen (siehe Harksen 2006) anschaut: Die Kunst besteht darin, einen sehr spezifïschen Habitus darstellen zu können, den man in der Regel nur über langjälurige Sozialisation erwerben kann. ${ }^{19}$

Aber abgesehen von solchen Krisensituationen sind Mitglieder von Professionen, so die These, vom Zwang zur übermäßig offensiven Kompetenzdarstellung befreit, weil sie sich zu einem nicht unerheblichen Teil auf die Kompe-

is Vgl. für eindrucksvolle Reaktionen won Professionellen bei K'risen aufgrund von Kiompetenzzweitein besonders anschaulich Moers 1990: 1 ?

19 Auch Buer würdigl die Darstellungsterstung Postels mit sehr treffsicheren Referiesungen der besonders aufschlussreichen Stellen aus dessen Buch, scheint dann aber nit der Aussage. dass „offensichtlich ..die Darstellungskompetenz oft viel entscheidender (ist) als die fachliche und "moralische Kompetenz, einer professioneilen Tätigkeit angemessen nachgehen zu können“ (Buer 2004: 172) einer inszenierungstheoretischen Interpretation zuzuneigen, die professionelles Handeln vorrangig als Darstellungskompetenz begreift. 
tenzvermutungen der Klienten gegenüber Professionen stützen können. Um den Gedanken zusammenfassend noch einmal zuzuspitzen: Professionen entlasten die Leistungserbringer davon, ihre Kompetenz zu inszenieren. Die Kompetenzvermutung der Klienten gegenüber Professionen reduziert für die Leistungserbringer die Notwendigkeit, ihre Kompetenz offensiv darzustellen und dadurch in das Problem des Selbstlobs zu geraten.

\section{Zum Zusammenhang von Kompetenz und Kompetenzdarstellung}

Dem dramatologischen Ansatz wird von Vertretern anderer Theorieschulen vorgeworfen, dass er sich zu viel mit Kompetenzdarstellung und zu wenig mit Kompetenzen auseinander setzt. Der Ansatz, so beispielsweise der Vorwurf von Vertretern der objektiven Hermeneutik, verkenne, dass es gerade bei professionălisierungsbedürttigen Tätigkeiten wie dem Recht, der Therapie, der Medizin oder der Religion nicht darauf ankomme, Kompetenzen darzustellen, sondem die Probleme der Klienten kompetent zu bearbeiten.

Diese Kritik am dramatologischen Ansatz ist sicherlich berechtigt, aber ein Aspekt wird aus meiner Sicht übersehen. Häut̆g ist es erst die Kompetenzvermutung des Klienten, die es den Leistungserbringern ermöglicht, den Arbeitsrahmen zu kontrollieren. Und erst durch die Kontrolle des Arbeitsrahmens können sie nach den eigenen Standards "guter Arbeit" wirken. Schon frühe Studien über so unterschiediiche Arbeitsfelder wie Restaurants, Schnellzüge und Psychiatrien haben gezeigl, dass der Erfolg eines Arbeitsverlaufs davon abhängt. dass der Angestellte die Initiative ergreift: Der Kellner vernittelt Kunden zwar das Gefühl, dass er ihnen zu Diensten steht, aber er strebt danach, die Kontrolle über den Arbeitsablauf zu behalten (vgl. Whyte 1949). Die Zugbegleiterin bietet zwar dem Kunden ihre Serviceleistungen in Form von Zugausküntten oder Kaffee an, aber sie lässt keinen Zweifel daran, dass sie die Oberhand behält (vgl. Heind 2005). In der Psychiatrie verlangt zwar das Krankheitsbild der Klienten. dass die Psychiater auf Feindseligkeit mit Wärme und Verbindiichkeit reagieren, aber gleichzeitig sind sie darauf angewiesen, die Arbeitssituation komplett zu kontrollieren (vgl. Goffnan 1956).

Eine solche Kompetenzvermutung des Klienten muss gerade in der Anbahnungsphase mobilisiert werden. Erving Goffman (1983:15) hat darauf hingewiesen. wie wichtig der Erstkontakt gerade in Interaktionen zwischen Professionellen und Klienten ist. ${ }^{20}$ Besonders deutlich wird dies bei Dienstleistern, die es mit

20 Dieser Ansatz ist jedoch mt der These ins Absurde übertrieben worden, dass der Erfolg einer Interaktion weitgehend von .ersten Eindruck“ abhăngl (siehe zu einer psychologischen Auseinandersetzung Bergler/Hoff 2001), einer eher widerständigen Klientel zu tun haben. Viele Lehrer nähmen, so Goffman, der Standpunkt ein, dass es zentral sei, ,, von Anfang an den richtigen Eindruck zu machen". Man müsse von Anfang an zeigen, wer der "Stärkere“" ist, sonst könte man gar nicht erst seine Kompetenzen darstellen. Bei manchen Wärtern in Nervenheilanstalten sei es, so Goffman, eine feste Überzeugung. dass man dem Patienten gleich am ersten Tag klar machen müsse, wer ,der Her im Haus ist". Damit würde man später viele Schwierigkeiten vermeiden. Aber auch bei Dienstleistem, die es mit einer Klientel zu tun haben, die freiwillig kommt und auch bereit ist, für die Dienstleistung zu bezahlen, gibt es die Notwendigkeit, in einem sehr frühen Stadium beim Klienten eine Kompetenzvermutung gegenüber dem Dienstleister zu erzeugen.

Ist das Arbeitsbündnis zwischen Leistungserbringer und Klient erst einmal etabliert, reduziert sich dieses Problem der Kompetenzvermutung erheblich. Genauso wie ein Patient, der über längere Zeit in der Behandlung durch eine Ärtin ist, sich irgendwann nicht mehr die Frage nach deren Kompetenz stellt, wird es auch einem Klienten gehen, der nach einer Reihe von Beratungssitzun. gen die Kompetenzvermutungen auf der Basis von als erfolgreich wahrgenommenen Supervisions- und Coachingsitzungen aufgebaut hat und aufgrund der gemeinsamen Interaktionsgeschichte mit Kompetenzvermutungen an die Beratung herangeht.

\section{Literatur}

Abagnale, Frank W./Redding, Stan $(2000)$ : Catch me if you Can: the Amazing True Story of the Youngest and most Daring Con Man in the History of Fun and Profit. New York, NY: Broadway Books.

Allensbacher Institut für Demoskopie (2005): Allensbacher Berufsprestige-Skala 2005. Allensback: Aliensbactier Berichte 12/2005.

Bales, Robert F. (1951): Interaction Process Analysis. A Method for the Study of Small Groups. Cambridge, MA: Addison-Wesley.

Bartens, Werner (2007): Arztkittel vor dem Aus. Der Arztkittel, die letzte Bastion des ärztlichen Standesbewasstseins, gerát in Gefahr: Der britische Gesundheitsminister will das Kleidungsstück abschaffen - weil ès zu unhygienisch ist. In: Süddeutsche Zeitung, 18.9.2007, S. 12.

Bergler, Reinhold/Hoff, Tanja (2001): Psychologie des ersten Eindrucks, Kölin: Deutscher Institutsverlag.

Buer, Ferdinand (2004): Über die professionelie Kompetenz, Professionalität kompetent darzuslellen. Und welche Rolle die Supervision heute dabei spielt. In: Buer, Ferdinand/Siller, Gertrud (Hg.): Die flexible Supervision. Herausforderungen - Konzepte - Perspekliven. Wiesbaden: VS-Verlag, S. 161-202. 
Clark, Timothy (1995): Managing consultants: Consultancy as the managentent of impressions. Buckinghan: Open University Press

Clark, Timothy/Salaman, Graeme (1996a): Teiling Tales: Management Consuttancy as the Art of Story Telling. in: David, Grani/Oswick, Cliff (Hg): Meraphor and Organizations. London: Sage, S. $167+184$

Clark, Timothy/Salaman, Graeme (1996b): The Management Guru as Organizational Witchdoctor. In: Organization. Jg. 3. S. 85-107.

Etzioni, Amitai (1965): Dual Leadership in Complex Oryanization. In: American Sociological Review, Jg. 30, S. 688-698.

Goethe, Johann Wolfgang von (1999): Torquato Tasso. In: Goethe, Johann Wolfgang von (Hg.) Poetische Werke, Band S. Essen: Phaidon Verlag. S. $613-697$

Goffinan, Erving (1956): The Nature of Deference and Demeanor. In: American Authropologist, yg 58, S. 473-502.

Goffman, Erving (1983): Wir alte spielen Theater. Die Selbstdarstellung im Allag. München et. al. Piper.

Goffman, Erving (1986): Techniken der Imageptlege, In: Goffrnart, Erving (Hg.): Interaktionsrituale. Frankfurt a.M.: Suhrkamp, S.10-54.

Guppy, Neil/Goyder, John C. (1984): Consensus on Occupational Prestige: A Reassessment of the Evidence. [n: Social Forces, Jg. 62, S. 709-725

Harksen, Jürgen (2006): Wie ich den Reichen ihr Geld abnahm. Die Karriere eines Hochstaplers. Frankfurt a.M.: Scherz-Veriag.

Heindl. Regina (2005): Dienstleistungsinteraktion in Zügen. München: Diplonarbeit Universitä München

Hitzler, Ronaid (1991): Goffmans Perspektive. Notizen zum dramatologischen Ansatz. In: Sozial wissenschaftliche informationen. H. 4/1991, S. 276-281.

Hitzier, Ronald (1992): Der Goffmenseh. In: Soziale Welt, Jg. 44, S. $449-461$.

Hodge, Robert W./Siegel: Paul M./Rossi, Peter H. (1964): Occupational Prestige in the United States, 1925-63. In: American Journal of Sxciology, Jy. 70, S. 286-302.

Hülsmann, Dorothea (1969). Schriftstellernde Betrüger. München: Dissertation aus der Nervenklinik der Universitäl München.

Ikusaka, Masatomi et al. (1999): Patients' Attitudes toward Consultations by a Physician Without a White Coat in Japan. In: Intental Medicine, Jg. 38: S. 533-536.

Kieserling, André (1999): Konımunikation unter Anwesenden. Studien über Interaktionssysteme. Frankfurt a.M.: Suhrkamp.

Kieserling, André (2004): Sechs Vorlesungen über Schichtung. Mainz: unveröff. Ms

Kriwy, Peter/Auınüller, Heimut (2007): Präterenzen von Patienten bei der Hausarzt- oder Krankenhaussuche. In: Das Gesundheitswesen, H. 8/9/2007, S. 464-469.

Kühl, Stefan (2008a): Die verflixte Sache mit der Kompetenzdarstellung. In: Schimank, Uwe; Nadine Schöneck (Hg.): Einładung zur Soziologie. Frankfurt a.M.; New York: Campus, erscheint Ende 2008.

Kühl, Stefan (2008b): Coaching und Supervision. Zur personenorientierten Beratung in Organisationen. Wiesbaden: VS-Verlag.

Luhmann, Niklas (1964): Funktionen und Folgen formaler Organisation. Berlin: Duncker \& Hunblot.

Luhmann. Niklas (1972): Die Organisierbarket von Religionen und Kirchen. In; Wössner, Jakobus ( $\mathrm{Hg}$.): Religion in Umbruch. Soziologische Beittäge zur Situation von Religion und Kirche in der gegenwärtiger Gesellschaft. Stuttgart: Ferdinand Enke, S. 245-285.

Luhınann, Niklas (1974): Professionelle Arbeit. Bielefeld: unveröff. Ms.

Luhmann. Niklas (1983): Legitimatıon durch Verfahren. Frankfurt a.M.: Suhrkamp.

Luhmant, Niklas (1997): Die Gesellschaft der Geseltschaft. Frankfurt a.M.: Subrkamp.

Lufmann, Niklas (2000): Organisation und Entscheidung. Opladen: WDV.
Mangham, lain L. (1978): interaction and Interventions in Organizations. Chichester: Wiley,

Mangham, lain L. (1986): Power and Performance in Organizations: an Exploration of Executive Process. Oxford: Basil Blackwell.

Mangham, Jain L.fOverington, Michael A. (1987): Organizations as Theatre. A Social Psychology of dramatic Appearances. Chicester: Wiley.

Marcus, Philip M. (1960): Expressive and Instrumental Groups. Toward a Theory of Group Structure. In: American Journal of Sociology, Jg. 66, S. 54-59.

Moers, Waiter (1990): Kleines Arschloch. Frankfurt a.M. Eichborn.

Murphy, Clare (2007): Death of the Doctor's White Coat . in: BBC News, 17.11.2007

Nakao, Keiko (1992): Occupational Prestige. In: Borgatta, Edgar F./Borgatla, Marie L. (Hg.): Ency. clopedia of Sociology, Bd. 3. New York: MacMilian. S. 1388-1391.

Neuberger, Oswald (1990): Der Witz der Arbeit. Bemerkungen zur Lache in den Betrieben. In Kunkel, Klaus (Hg.): Nichts zu lachen? Des Wohlstands siî-saure Gemütslage. Köln: Informedia, S. $51-130$.

Pfadenhauer, Michaela (1999): Rollenkompetenz. Träger, Spieler und Professionelle ais Akteure für die hermeneutische Wissenssoziologie. In: Hitzler, Ronald; Jo Reichertz, Jo; Norbert Schröer (Hg.): Hermeneutische Wissenssoziologie. Standpunkte zur Theorie der Interpretation. Konstan 2 UVK, S. 267-285.

Pfadenhauer, Michaela (2003): Professionalität. Opladen: Leske + Budtich.

Pomerantz, Anita (1978): Compliment Responses: Notes on the Co-operation of Muitiple Constraints. In: Schenkein, Jim (Hg.): Studies in the Organization of Conversational Interaction New York: Academic Press, S. 79.112

Postel, Gert (2001): Doktorspiele. Geständnisse eines Hochstaplers. Frankfurt a M.: Eichborn.

Tedeschi, James T. (1981): Impression Management Theory and Social Psycholocial Research. New York, NY: Academic Press

Whyte, Willian H. (1949). The Social Structure of the Restaurant It. Anterican Jounal of Sociology, Jg. 54, S. 302-310.

Xueguang, Zhou (2005): The institutional Logic of Occupational Prestige Ranking: Reconceptuatization and Reanalyses. In: The American Joumal of Sociology, Jg. 111, S. 90-140.

Zuckmayer, Cari (1995): Der Hauptmann von Köpenick. 74. Auft. Frankfust a.M.: Fischer. 